\title{
Fenomenología
}

\section{La historia de enfermería: trascendencia del fenómeno cuidado y su relación con el tiempo}

Nursing history: transcendence of care phenomenon and its relationship with time

\section{História da enfermagem: importância do fenômeno cuidados e sua relação com o tempo}

Javier Badillo Zúñiga ${ }^{1}$; Ana R. Hernández Morales²; Alfredo Bermúdez González ${ }^{3}$

${ }^{1}$ Alumno de la $10^{a}$ Generación del Programa de Maestría en Enfermería, UNAM. javier_7badillo@yahoo.com.mx, cel. (55) 27329549

${ }^{2}$ Alumna de la 10a Generación del Programa de Maestría en Enfermería, UNAM. anyshdzm@gmail.com

${ }^{3}$ Maestro de Seminario de Epistemología y Enfermería y tutor del PME, UNAM. kandisky56@hotmail.com

Cómo citar este artículo en edición digital: Badillo Zúñiga, J; Hernández Morales A.R; Bermúdez González, A. (2013) La historia de enfermería: trascendencia del fenómeno cuidado y su relación con el tiempo. Cultura de los Cuidados (Edición digital)17, 36. Disponible en: <http://dx.doi.org/10.7184/cuid.2013.36.02>

Correspondencia: Alfredo Bermúdez González. Escuela Nacional de Enfermería y Obstetricia. Unidad de Investigación.

Cubículo 1. México. D.F. tel. 56552332 ext. 309, Área secretarial 281

Correo electrónico: kandisky56@hotmail.com

Recibido 20/3/2013. Aceptado 11/05/2013

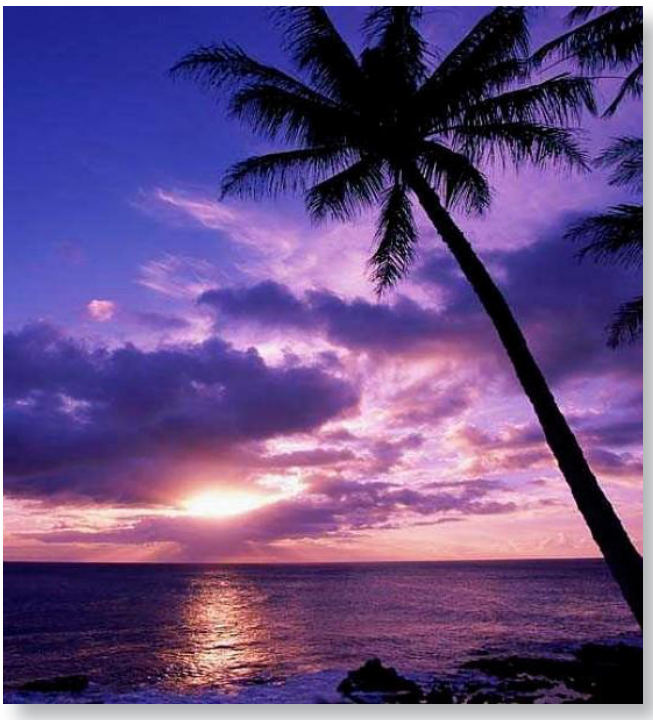

ABSTRACT

History is a discipline that contributes to the nursing to trace the significance of their professional work, caring. The close relationship with time reminds us of Martin Heidegger as the philosopher who raises an inseparable articulation between time and the being through caring. As we reflect on the philosophical context of caring and its relation to time, which identifies a paradox in nursing professional work, who are serving schedules, routines and activities outside the ethical issue of giving one point for each thing affected by accelerated dynamics, overcrowding of patients in health institutions, making it impossible to provide care as such, as its temporality is to meet the needs for the existence of the person from allowing conceptualize care as a whole being and in that sense, have the time required to perform satisfactorily quality care and encourage the process the construction of discipline.

Key words: history, nurses, care and time. 


\section{RESUMO}

A História é uma disciplina que contribui para a enfermaria para traçar o significado da sua vida profissional, atencioso. A estreita ligação com o tempo nos faz lembrar de Martin Heidegger como o filósofo que levanta uma ligação inseparável entre o tempo e sendo por carinho. Ao refletirmos sobre o contexto filosófico do cuidado e sua relação com o tempo, o que identifica um paradoxo em enfermagem do trabalho profissional, que estão servindo horários, rotinas e atividades fora a questão ética de dar um ponto para cada coisa afetada pela dinâmica acelerada, a superlotação de pacientes em instituições de saúde, o que torna impossível a prestação de cuidados como tal, sua temporalidade é para atender às necessidades para a existência da pessoa de permitir que o cuidado conceituar como um todo, sendo e, nesse sentido, têm o tempo necessário para realizar atendimento de forma satisfatória a qualidade e incentivar o processo de construção de disciplina.

Palavras-chave: história, enfermeiros, cuidado e tempo.

\section{RESUMEN}

La historia es una disciplina que contribuye a la enfermería para dejar huella de la trascendencia de su quehacer profesional, el cuidado. La estrecha vinculación con el tiempo nos remite a Martin Heidegger como el filósofo que plantea una articulación inseparable entre el tiempo y el ser a través del cuidado. Por lo que se reflexiona sobre el contexto filosófico del cuidado y su relación con el tiempo, que permite identificar una paradoja en el quehacer profesional de enfermería, quienes se encuentran cumpliendo horarios, rutinas $y$ actividades fuera de la cuestión ética de otorgar un momento para cada cosa, afectado por aceleradas dinámicas, sobrepoblación de pacientes en las instituciones de salud, haciendo imposible proporcionar el cuidado como tal, ya que su temporalidad consiste en satisfacer las necesidades para la existencia de la persona a partir de cuidados que permitan conceptualizarlo como un ser completo y en ese sentido, contar con los tiempos necesarios para poder realizar de manera satisfactoria un cuidado con calidad y fomentar el proceso de la construcción de la disciplina.

Palabras clave: historia, enfermeria, cuidado y tiempo.

\section{INTRODUCCIÓN}

El cuidado está considerado como un proceso de vertebración en la disciplina de enfermería y el tiempo como su principal limitante y posibilitador para la satisfacción de las necesidades del cuidado del ser. Esta ambivalencia se ve reflejada en la práctica clínica de la enfermería, donde además se adjudica actividades ajenas al cuidado del ser, desviando su praxis con repercusión ética.

Considerar a la persona desde la dinámica de un cuerpo social, que al transcurrir en el tiempo va creando la interrelación con su entorno, es un detonador que genera nuevas necesidades por satisfacer, tal como señala Agnes Heller (1998), citando a Marx cuando dice: La diversa conformación de la vida material depende en cada caso, naturalmente, de las necesidades ya desarrolladas y tanto la creación como la satisfacción de estas necesidades es de suyo, un proceso histórico*. La historicidad del ser tiene necesidades propias derivadas de sí mismo y de su interacción con los demás. La enfermería es una disciplina que realiza cuidados para la satisfacción de necesidades de la salud y la 
enfermedad, abordando aspectos anímicos de la persona. La interrelación que se genera en la satisfacción de necesidades es postulada por Heller, señalando que el objeto más elevado de la necesidad humana es el otro hombre, lo que permite ver la compleja interrelación (persona/ enfermero(a)/ tiempo), de ahí la importancia de una reflexión sobre una intuición del tiempo como una aportación para la enfermería desde la investigación histórica.

Con respecto a la interrelación de la enfermería y la persona para la satisfacción de necesidades del hombre se debe reflexionar en la visión de la teoría de Marx cuando escribe que:

La necesidad se refiere en todo momento a algún objeto material o a una actividad concreta. El primer hecho histórico es por consiguiente, la producción de los medios indispensables para la satisfacción de estas necesidades... En la producción de los instrumentos aptos para satisfacer las necesidades fundamentales, la necesidad de los instrumentos es ya una necesidad nueva. La necesidad humana se realiza, en el proceso de objetualización; los objetos $<<$ dirigen $>>y<<$ regulan $>>$ al hombre en el desarrollo de la necesidades respectivas. La capacidad, la actividad concreta es por consiguiente una de las mayores necesidades del hombre. ...dentro de esta estructura de necesidades predominaba la calidad y no la cantidad., Carlos Marx, (1968).

Desde dicha perspectiva el hecho histórico de la creación de la enfermería, se da a partir de un proceso de objetualización de una actividad concreta, la satisfacción de necesidades con el cuidado. La enfermería entonces es un producto y productor de medios para la satisfacción de las necesidades

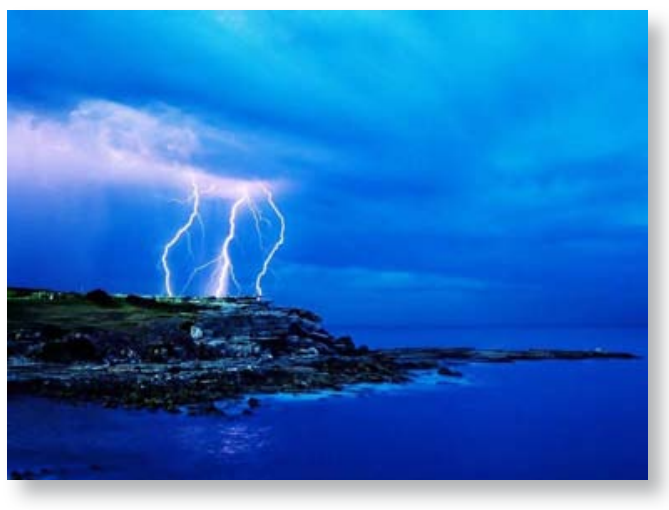

de la salud, que debe dirigir y regular con el cuidado, haciendo predominar la calidad.

La satisfacción de las necesidades de salud es un elemento indispensable de la calidad del cuidado, surge en 1860 con Florence Nightingale, siendo pionera en el control de la calidad de los cuidados de enfermería de los hospitales militares británicos, refiere en sus escritos que el ambiente físico del paciente es de capital importancia en la calidad asistencial, refiere Zárate (2011).

Al reflexionar sobre lo anterior, se puede decir que históricamente enfermería ha realizado su quehacer profesional cumpliendo con horarios, rutinas y actividades establecidas fuera del contexto del cuidado de calidad, lo cual puede verse en contraposición a la cuestión ética de otorgar un momento para cada cosa, cuando ya no es posible proporcionar una atención con total calidad, ya que su temporalidad consiste en ubicar la esencia de la persona en tiempo, lugar y espacio conceptualizándolo como un ser completo; Ferrer (2011) retoma lo postulado por Edith Stein para mencionar que la persona es: ...en cuanto yo, que abarca el cuerpo y el alma, ilumina por medio del entendimiento $y$ domina por medio de la voluntad como soporte levantado por detrás y por encima de todo lo corporal-anímico o como forma de plenitud que lo une todo ${ }^{* *}$, dichas características planteadas 
por Stein constituyen un ser complejo que desde tal plenitud, los fenómenos de salud y enfermedad que vive, cada momento se torna distinto, resaltando así la necesidad de dar un tiempo específico para el cuidado enfermero.

De tal manera que Siles (2007), menciona que Heidegger concibe la cura como una entidad dispensadora de cuidados, que modela al ser durante todo su ciclo de vida (nacimiento hasta su muerte), manteniendo mesura entre pensamiento, valores y conducta al satisfacer las necesidades del ser mediante el cuidado a lo largo del tiempo, cuyo término visto bajo esta perspectiva, debe ser objeto para la construcción del conocimiento en enfermería desde tales núcleos paradigmáticos.

Cabe señalar que es fundamental contar con los tiempos necesarios para poder realizar de manera satisfactoria el cuidado de enfermería con calidad y fomentar el proceso de la estructura en la construcción de la disciplina, ya que si consideramos que el tiempo en la práctica clínica es de vital importancia para el ser, que se requiere para satisfacer sus necesidades, por lo tanto, es a priori determinar una regulación institucional desde la perspectiva de enfermería para la disciplina.

Actualmente menciona Zárate (2011), que Avedis Donavedian define la calidad de la atención en salud en dos dimensiones: la técnica que se refiere a la aplicación de la ciencia y la tecnología y; la interpersonal que incluye los valores y normas socialmente definidas que gobiernan la interacción de los individuos en general o de forma particular.

En la experiencia real de la práctica de enfermería, se enfatiza la dimensión técnica por encima de la dimensión interpersonal quedando ésta última, relegada; si bien el cuidado es el quehacer de enfermería, entonces la respuesta a las necesidades de la persona deben encaminarse según Zárate (2011), hacia un cuidado de calidad que sea seguro, efectivo, centrado en el paciente, oportuno, eficiente $y$ equitativo.

Es necesario que enfermería busque continuamente estrategias en el ámbito clínico y comunitario que fundamenten una mejor calidad del cuidado dirigidas al bienestar de las personas y a la comprensión de los procesos vitales de la salud y la enfermedad.

\section{OBJETIVOS}

- Realizar un análisis hermenéutico de la relación entre el cuidado y el tiempo y su importancia histórico-filosófico

- Mostrar la importancia de la investigación histórica-filosófico como fuente subyacente de y para la epistemología en enfermería, desde el discurso de los documentos analizados para la mejora de la calidad de la atención

- Establecer el contexto de la responsabilidad en el cuidado del ser humano y su proceso histórico, a partir del análisis, como un fenómeno recurrente en el ejercicio profesional de enfermeria

\section{METODOLOGÍA}

El presente trabajo se realizó bajo el análisis del método histórico-filosófico con la hermenéutica, ya que el objetivo de la revisión documental requiere de la interpretación de los datos que permita mostrar la reflexión del discurso de los artículos abordados, basado en el conocimiento previamente adquirido en el seminario de Epistemología y Enfermería que forma parte del programa de maestría, así como de diversas fuentes documentales consultadas. Estos datos, son expuestos desde el análisis hermenéutico de la información seleccionada, para aprender y aprehender el fenómeno, mismo que pretende ir cobrando 
un sentido para la integración de los aspectos que desde el planteamiento de la hermenéutica postulada por Heidegger, debe contemplarse la visión de la existencia del fenómeno y sus significaciones para su comprensión científica.

\section{Tratamiento metodológico}

1.- Selección de fuentes

2.- Revisión documental

3.- Selección de información

5.- Análisis del discurso

6.- Interpretación de los datos

\section{DESARROLLO}

Se considera al cuidado como la columna vertebral de enfermería y el tiempo cronológico, entendido como los filósofos griegos emplearon el término "Crhonos" para referirse al tiempo cronológico, al origen fenomenológico del cuidado y la importancia del concepto de tiempo en la historia de la enfermería, como su principal limitante; en tanto que, el concepto de momento para cada cosa (káiros) es para identificar la importancia del momento vivido oportunamente (Siles y Solano, 2007), entendido a su vez, como posibilitador de la permanencia para la satisfacción de las necesidades de la persona. Desde el punto de vista filosófico definir el tiempo nos remite al constructo: ser con los demás y va más allá de la medición física de la acción, transformación constante, incluso rebasa la presencia secuencial de eventos naturales como el día y la noche. El tiempo, entonces, se refiere al movimiento del ser humano creando relaciones de unos con otros; al respecto escribe Edith Stein que: no se vive sólo objetivamente en la comunidad popular, sino que se recibe de ella la formalización típica $y$ se desempeñan funciones en ella: es preciso tener la noción de pueblo como tal y saber de la propia pertenencia al mismo..., (Ferrer, 2011), Los profesionales de enfermería a través de diversas gestiones, han buscado la forma de otorgar cuidados de calidad a pesar de la gran demanda que existe de los servicios de salud a nivel mundial y de los cortos tiempos que tienen para dar cuidado a las personas que están bajo su responsabilidad; por lo que Stein remite a pensar en un trabajo institucional colectivo, grupal, con el objetivo del dominio de la praxis, en la que se contemple la dinámica del cuerpo social, la persona, y que genere cuidados de calidad. Un ejemplo y oportunidad para llevar a cabo este trabajo grupal, son la implementación colectiva $y$ general de los indicadores de calidad, tales como la prevención de ulceras por presión, las medidas de seguridad personal en pacientes hospitalizados, entre otros múltiples cuidados, colocando a la persona inmersa en la dinámica de un cuerpo social, interrelacionado con su entorno, el cual le genera nuevas necesidades por satisfacer. A propósito de ello y a modo de énfasis, Marx plantea que: La diversa conformación de la vida material depende en cada caso, naturalmente, de las necesidades ya desarrolladas y tanto la creación como la satisfacción de estas necesidades es de suyo, un proceso histórico... (Agnes Héller, 1998).

A través del tiempo, las enfermeras han desarrollado la práctica institucional del cuidado con horarios y rutinas establecidas que se contraponen a la cuestión ética de otorgar el espacio y el tiempo requeridos para promover el equilibrio entre los diferentes elementos del paradigma de enfermería: salud, entorno, cuidado y persona. Kuhn desde su percepción de ...paradigma donde se aproxima una comprensión histórica de los cambios producidos en la ciencia a través de los tiempos, (Toro, 2005), es un referente para considerar 
que desde el paradigma de enfermeria es posible hacer las reflexiones actuales sobre su propia práctica.

En el acontecimiento histórico se determina al ser como un ente desde su concepción hasta más allá de su muerte; su temporalidad consiste en ubicar la esencia del individuo en tiempo, lugar y espacio, conceptualizándolo no sólo como algo cognoscente, sino como un ser completo (corpóreo - anímico - espiritual) ubicándolo en las dimensiones del haber sido, el devenir y el actual, generando así la conceptualización de enfermería desde la naturaleza misma del ser humano.

Desde que el hombre toma consciencia de su ser como parte de un universo, también conoce su temporalidad, su finitud, y en el intermedio de ese principio y fin, una infinita gama de posibilidades. En relación con el enunciado anterior, la teoría de las necesidades (Héller, 1998), establece que el objeto más elevado de la necesidad humana es el otro hombre, debido a ello, se explica la relación persona/enfermero(a)/tiempo ocupándose incluso de la proporción de cuidados para una muerte digna.

Es preciso entonces, reflexionar sobre dar valor al instante, (káiros), con respecto al tiempo, (Chronos), como una aportación para la enfermería desde la investigación históricofilosófica. Dicha historicidad del ser, no sólo se ubica en el tiempo vulgar, sino en el tiempo espiritual de manera contextual, individual y colectiva donde el hombre permanece con su esencia en la naturaleza de su ser.

En este sentido se identifica que el ser humano ha desarrollado conceptos que le sirven como eje rector de su existencia, uno de ellos es el tiempo, y es en la enfermería donde adquiere una dimensión especial, ya que a través del quehacer específico del cuidado enfermero, le

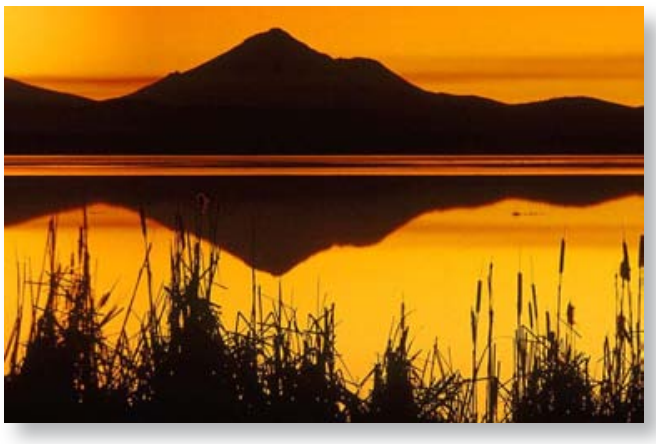

corresponde a sus profesionales ser testigos y partícipes del momento en que se inicia y finaliza el tiempo de las personas: el nacimiento y la muerte, proporcionando cuidados específicos en el devenir de la vida y su final.

A su vez, las convivencias sociales favorecen la adquisición de conocimientos para cuidar de la mejor manera a uno mismo y a los demás, como se ha realizado desde épocas remotas, aprendemos cuidando, pasando de generación en generación; un claro ejemplo es la muerte, fenómeno constante en la práctica enfermera; tal como afirma Heidegger: la muerte es un fenómeno y una propiedad de la vida, (Cataldo, 2003). Ocupando un instante irrepetible donde la enfermería debe otorgar los cuidados a la persona en el trance de sus últimos momentos, obedeciendo al ideal que propone Heidegger sobre la libertad para la muerte: "el abandonarse a la no existencia., (Cataldo, 2003)" Contrario a nuestra cultura, donde no se acepta la cotidianidad de la muerte como una etapa de la vida para trascender; al respecto afirma Schopenhauer, que el tiempo no forma parte de las cosas en sí, es fenoménico y superficial.

¿No es desolación y vacío lo que recibiría al niño en el momento de nacer si la provisión de cuidados no le brindara la posibilidad de continuar vivo? ¿No es de la desolación y el vacío de lo que necesita ser rescatado el que está muriendo, el que ya casi no es en el tiempo? Se 
puede inferir que ante tales fenómenos que provoca vivir la vida ha dado pie al cuidado como hecho histórico, por lo que no es exclusividad de la enfermería. Sin embargo, Nightingale reflexiona en este discurrir histórico cuando dice que cualquier persona “en algún momento de su vida. . . tiene a su cargo la salud de alguien...(Nightingale, 1991).

El proceso histórico del propio fenómeno de cuidar a quienes no pueden hacerlo por sí mismos, ha dado pie a la evolución hacia una mayor complejidad de cuidados, dando estructuras de identidad a la enfermería con el cuidado, que tiene como objetivo, la sobrevivencia y el estado de bienestar de aquellos que incluso, están por perecer.

Ahora que la enfermería se ha definido en el fenómeno de cuidar y lo ha convertido en una actividad profesional, se guía a través de los principios bioéticos que rigen su actividad ante aquellos que requieren de apoyo. La necesidad del cuidado es la que mantiene una estrecha unión entre los miembros de una sociedad. Identificarnos como seres que necesitamos del próximo y que éste nos necesita requiere de una introspección en la que se delimite los alcances de su significado y de su trascendencia. Las enfermeras son las profesionales, las expertas en cuidar; a propósito del momento histórico y los esquemas de relación social basados en la economía, la actividad del cuidado debería ser encuadrada con la importancia que se merece, demostrando con ello que el cuidado se hace visible en beneficio de la sociedad.

Cabe señalar que ante el contexto de un cuidado encarecido por los sistemas de salud, es fundamental contar con los conocimientos necesarios para poder realizar de manera satisfactoria, efectiva, un cuidado con calidad, actitud positiva, fomentando la interacción con el ser que requiere del cuidado en naturaleza de la vida.

Puede afirmarse que el ser humano ha desarrollado conceptos que le sirven como eje rector de su existencia, su permanencia en el tiempo, aquello que le va ha permitir esa permanencia en el tiempo, el cuidado. La profundidaddelfenómenoabarcaexplicaciones o respuestas teleológicas, filosóficas o estéticas, como en el reflexivo poema, Nocturno muerte. Quién medirá el espacio, quién me dirá el momento en que funda el hielo de mi cuerpo y consuma el corazón inmóvil como la llama fría... (Xavier Villaurrutia, 1933), esto es un reflejo estético-poético del cuestionamiento ontológico que inquieta al humano: el tiempo y su permanencia en este mundo a través del cuidado.

\section{CONCLUSIÓN}

Abarcando el aspecto teleológico y estético, a partir de Heidegger y otras perspectivas filosóficas, se analizaron los términos de ontología fenomenológica del ser y tiempo; reflexionando sobre la existencia del Sein, sus actos (cuidado) y su relación con el mundo (lo que podría adecuarse a enfermería). La necesidad del saber estar ahí con sus bases teóricas orientadas al cuidado convirtiéndose en un proceso dinámico y temporal, ubica al profesional de enfermería con la persona bajo un contexto sociocultural donde ejecuta una acción mediante un conocimiento interactivo que conlleva a la enfermería a madurar bajo experiencias previas ante el cuidado individual y colectivo; convirtiéndose en un proceso objetivo manteniendo mesura entre pensamiento, valores y conducta para satisfacer las necesidades del ser mediante el cuidado.

No se debe dejar de lado el contexto filosófico de la persona ninguno de los 
profesionales de enfermería, ya que esta persona se encuentra bajo un contexto de necesidades. Se trata de personas que existen, que viven, que necesitan algo o de alguien, que históricamente requiere de cuidado. Es decir; que están ahí, frente a nosotros, junto a nosotros, en el tiempo.

Hegel dice que en el sentir somos la unidad en la diferencia, se entiende entonces que es necesario establecer una clara distinción reflexiva en relación al sujeto-objeto; por otro lado, Aristóteles quiere decir ...mi acto de percibir no es ni mi acto de ser ni el acto de ser del originante de mi percepción,... (Alvira, 1979). No debemos perder de vista que nuestra relación con el sujeto de estudio se basa en la percepción que tenemos del objeto como ser inmerso en el contexto y tiempo en el que se lleve a cabo dicho cuidado.

Estar ahí, es estar en el mundo, en nuestro mundo profesional y humano. El ser humano es proyección con todo lo que contribuye a lograr la felicidad humana, aspiraciones para el cuidado de la persona, el cuidado fundamental de nuestra existencia y de la existencia de los demás.

En el presente trabajo contribuyeron todos los alumnos del grupo del Seminario de Epistemología y Enfermería del Programa de Maestría en Enfermería.

\section{REFERENCIAS BIBLIOGRÁFICAS}

- Alvira, R. (1979) Reflexiones sobre el concepto de percepción en la filosofía Aristotélica. VI congreso nacional de Psicología Pamplona. Pamplona.

- Blog: Filosofía e historia. (2009) El tiempo según Martín Heidegger. Concepto del tiempo. Activa en: http://filosofiaehistoricidad.blogspot.mx/2009/04/el-tiempo-segun-martin-heidegger.html, [Consulta: 2 Marzo, 2012]

- Cataldo, S. G. (2003) Muerte y libertad en Martin Heidegger. En Revista Philosophica No 26, Instituto de Filosofía Pontificia Universidad Católica de Valparaíso.
- Ferrer Santos, U. (2011) Edith Stein. Philosophica: Enciclopedia filosófica, Fernández Labastida, F. - Mercado, J.A. editores Activa en URL: http://www.philosophica. info/archivo/2011/voces/stein/Stein.html, [Consulta: 2 Marzo, 2012]

- Heller, A. (1998) Teoría de las necesidades en Marx, Grupo Martes, Activa en URL: http://grupomartesweb. com.ar/index.php? option $=$ com_content $\& v i e w=\operatorname{arti}$ cle\&id=152:agnes-heller-teoria-de-las-necesidadesen-marx \&catid $=45$ :textos-general $\&$ Itemid $=58 \# \_f t n 1$, [Consulta: 1 de marzo, 2012]

- Lorca Gómez, O. (2002) Al hilo de una lectura del 19 de Los problemas fundamentales de la fenomenología de Martín Heidegger, Tesina para grado de Lic. en filosofía. Un acercamiento a la temporalidad. Facultad de filosofía y humanidades, Universidad de Chile. 2002.

- Marx, C. (1968) La ideología alemana. Pueblos Unidos. Montevideo, Argentina.

- Nightingale, F. (1991) Notas sobre enfermería, Salvat, Bacelona.

- Pifarré Ll. (2007) Heidegger y la pregunta por el ser. El retorno al fundamento. Biblioteca católica digital. Activa en URL: http://www.conocereisdeverdad.org/website/ index.php?id=3386, [Consulta: 2 Marzo. 2012)

- Siles, J; Solano R. C. (2007) El origen fenomenológico del "cuidado" y la importancia del concepto de tiempo en la historia de la enfermería. Cultura de los cuidados. 11(21):19- 26

- Toro, A; Marcano, L. (2003) La categoría paradigma en investigación social. Activa en URL: http://www.saber. ula.ve/bitstream/123456789/21020/1/articulo1.pdf, [Consulta: 1 Marzo, 2012)

- Villaurrutia, X. (1933) Nocturno muerto. En: Nocturnos, Ed., Fábula. Miguel N. Lira, México.

- Zárate, G. R. y cols. (2011) La calidad y seguridad del paciente: elementos conceptuales. Enfermería y seguridad de los pacientes, Organización Panamericana de la Salud, Washington.

- Zárate, G., y cols. (2011) La calidad y seguridad del paciente: elementos conceptuales. Enfermería y seguridad de los pacientes, Organización Panamericana de la Salud, Washington. 